\title{
A Public Health Perspective on Preventing and Controlling the Spread of Coronavirus Disease 2019
}

\author{
Xifeng $\mathrm{Wu}^{1,2,3, *} ;$ Yuanqing $\mathrm{Ye}^{1,2,3}$
}

On March 11, 2020, the World Health Organization (WHO) formally declared coronavirus disease 2019 (COVID-19) a worldwide pandemic as the virus spreads rapidly with new cases and deaths rising exponentially in many countries. As of March 12 , there were 125,048 confirmed cases and 4,613 deaths, and the numbers are still surging affecting 118 countries (1). Now we know that regional efforts to contain individual outbreaks have failed. The next phase of epidemic control is mitigation, and China has implemented multiple effective measurements such as mandatory citywide lockdowns to isolate and block the spread since January 2020. Recent data has shown evidence of controlling the epidemic (2).

\section{How did the COVID-19 epidemic start?}

The exact origin of COVID-19 in humans has not been identified. This infectious disease is reported to have started in December 2019 from the Huanan Seafood Wholesale Market in Wuhan, Hubei Province of China. By December 31, 2019, officials in Wuhan confirmed dozens of pneumonia cases of unknown etiology. Epidemiologists and infectious disease experts from China CDC investigated the seafood market in the early days of January 2020 but the market had already been closed and cleaned. Due to the possibility of asymptomatic transmission of the virus, identifying patient zero is incredibly difficult. However, Chinese scientists quickly and successfully identified the genetic sequence of COVID-19 and reported the sequence on January 7, 2020 (3).

\section{Containing the spread of COVID-19 in China - lessons learned from Zhejiang Province and Hangzhou City}

A couple of days after the lockdown of Wuhan, the epicenter, on January 23, 2020, Zhejiang Province was the first provincial-level administrative division (PLAD) in the nation to raise the risk management response to the highest level. Hangzhou, the capital city of this province with a population of 11 million, started implementing extreme measures known as social distancing to contain the spread of COVID-19. From an epidemiological perspective, the benefits of stringent interventions are maximized if they are implemented early and maintained. Hangzhou has proven to be a perfect example of controlling the COVID-19 epidemic in a large metropolitan area (4). In controlling the COVID-19 outbreak in the early days of its emergence, the local officials often face significant challenges in making unprecedented decisions that will dramatically impact the economy and the social lives of millions of citizens. Timing also plays a key role in this decision-making process. The provincial and city authorities and experts from Hangzhou CDC worked closely to implement a series of actions (4):

Establishing a city-wide communication system to engage every individual to participate in implementing the city guidelines through apartments, communities, business, organizations, schools, and public facilities. This participation was major as it allowed the city, with the help of all involved, to successfully implement this mandatory lockdown for over a month. Strict entrance and exit control by local officials across the city were continuously maintained. Many citizens also monitored their neighbors. Sanitization measures were applied from every doorknob to every elevator keypad.

Maintaining food and supply flow through organized and government-controlled arrangements. Through online ordering and designated delivery groups, the sustained flow of fresh food and supplies were managed and maintained by district areas.

Reserving and designating infectious disease care and management hospital facilities to isolate, monitor, and treat COVID-19 positive patients. There are 22 hospitals in Hangzhou. The city designated two for COVID-19 patients and an additional 168 designated 
facilities (i.e., hotels) to isolate and monitor COVID19 positive but clinically mild symptom patients. In addition, there were 2,018 doctors and nurses from Zhejiang deployed to Wuhan to aid the response to the disease, among them, 318 were from Hangzhou city level hospitals and 525 were from affiliated hospitals of Zhejiang University.

Establishing electronic recording and tracking systems, and local response teams to continuously handle identified cases. Hangzhou implemented a big data analytics system and information technology that was named "one map, one QR code, and one index". The health QR codes are established for everyone in the city and those who enter the city. Green codes allow one to move freely in the city, yellow codes require 7-day self-quarantine, and red codes require 14-day self-quarantine. Yellow and red codes can turn green after completion of the quarantine periods. The health surveillance system also tracks self-monitored temperature data that is recorded twice a day. Hangzhou's CDC monitors the data.

As of March 12, 2020, there have been no new COVID-19 cases in Hangzhou for 22 days, and all 169 previously confirmed patients have been cured and discharged. The city is still under tight controls but started to allow workers to come back in phases based on priority with close monitoring and strict guidelines in place.

At the national level, the disease control and prevention measures were also implemented for most PLADs and cities. From a public health perspective, the national and local control strategies and measures have the following characteristics: mobilizing the whole nation at multiple levels from central government to individual families, responding (i.e., identification and detection of the virus) and implementing measures quickly; systematic and proactive risk management based on collaborations between government officials and health experts; the implementation of big data and information technology; and keeping the public well informed.

\section{Role of the public health system in controlling the COVID-19 epidemic}

China CDC is a governmental and national-level technical organization specialized in disease control and prevention. Under the leadership of the National Health Commission (NHC), China CDC exerts its function by providing technical guidance and support of public health. Unlike the US CDC which is part of a governmental agency, China CDC does not have executive authority to implement a wide range of measures in case of an emergency. Its role only allows the organization to report an epidemic and/or emergency to the government and participate in the preparation and response of public health emergencies as technical and scientific resources.

Following the SARS outbreak in 2003, China enacted two laws: The Regulation on Public Health Emergency and The Measures for the Administration of Information Reporting on Monitoring Public Health Emergencies and Epidemic Situation of Infectious Diseases (5-6). The government established a management system for public health emergencies and detailed the principal rules for the prevention and control of infectious disease (i.e., infection source control, interruption of route of transmission, and susceptible person's protection). Both central and local governments are in place to provide public health emergency responses (e.g., techniques, personnel, materials, and management preparedness), and an emergency information dissemination system that provides quick (within 2 hours), accurate, and comprehensive release of information. The public health management system reform led to better handling of an epidemic of infectious diseases. For example, the Chinese Ministry of Health $(\mathrm{CMH}$, the precursor to the NHC) issued a swine flu prevention guide on April 29, 2009, 12 days before the first reported H1N1 case in China (7). On April 3, 2013, 4 days after the first $\mathrm{H} 7 \mathrm{~N} 9$ confirmed case, the $\mathrm{CMH}$ also issued a nosocomial $\mathrm{H} 7 \mathrm{~N}$ 9-infection prevention guide (8).

It is worth noting that China's public health response and management system contributed significantly to handling the COVID-19 crisis after the outbreak. For example, the rapid publication of COVID-19 genetic sequence information allowed scientists across the globe to immediately start developing vaccines; peer-reviewed publications of epidemiological and clinical case analysis provided first-hand information for healthcare workers on how to detect, isolate, and treat the disease caused by COVID-19; and multiple guideline publications and case tracking information on COVID-19 greatly promoted public awareness of this disease. The COVID-19 epidemic in China showed a clear picture of what would happen if a potential human-to-human transmissible infectious source was left unattended in 
the community. However, other parts of China appeared to effectively mitigate the spread of the disease and many have shown significant improvements recently. Therefore, there is a need for the continuous improvement of the current public health management system. Specific strategies include the following:

To have more involvement of multidisciplinary experts (epidemiology, infectious disease, microbiology, clinical medicine, etc.) from CDCs, hospitals, and universities in the local and central government decision-making process.

To improve the emergency medical supply management system and plans to ensure clear pathways to follow in case of an epidemic emergency.

To establish a big data platform for disease control and prevention. On the premise of ensuring information security, colleges and universities cooperate with CDC to integrate basic population information, population movement information, patient information, medical insurance information, medical treatment information, and CDC data to establish a big data platform. This platform can realize the real-time, automatic, and quantitative reporting of new infectious diseases, and establish a system for prediction, early warning, and emergency response mechanisms to prevent the delayed detection and response to public health emergencies.

\section{Building a strong public health team to safeguard people's health}

In 2018, hospitals in China received a total budget of 269.7 billion RMB while CDC only received 51.1 billion (9). This is a clear reflection of the government's primary focus on treatment versus the prevention of diseases. In general, our public health talent cannot meet the needs of the "Healthy China 2030" in terms of quantity and quality. As of 2018 , there are 187,826 employees at all levels of CDCs in China and that is only $1.35 \mathrm{CDC}$ employees per 10,000 population, which is about one-fifth that of the United States (10). The overall quality of CDC employees is also a concern. Statistics show that only $44.2 \%$ of CDC health technicians have a bachelor's degree or higher. In addition, the lack of practical skills and knowledge of grassroots public health personnel needs to be improved. All of those are directly and indirectly linked to the current public health education system in China. Despite notable improvements in the higher education field, public health is still largely functioning as an auxiliary branch of a medical school. Significant reforms in our higher education system are the key to future success in building "Healthy China 2030":

Increasing the scale of training and scientific levels of public health: building public health colleges and universities; establishing key national public health research centers focusing on both major communicable and non-communicable diseases; and increasing governmental funds supporting schools and colleges of public health across the nation.

Optimizing the public health personnel training system: First, building a rigorous system to train leaders with high-level doctoral public health degrees. Second is to train multidisciplinary professionals of public health and general medicine. Establish a " $4+3$ " training mechanism, where a student studies medicine and public health courses in the first 4 years and continues with general medical practical training in the following 3 years. Preventive medicine students should also be trained in general medicine and be allowed to practice clinically after graduation.

Enhancing hands-on training in the public health professional curriculum: enhance practical trainings in various public health facilities for undergraduate and graduate students; increasing practical-skills-oriented courses for emerging infectious diseases in the undergraduate curriculum; improving practical skills training; strengthening the teaching of social practices with respect to emerging infectious diseases, and increasing training for public policy management and emergency responses.

Expanding the coverage of public health education: establishing a public health knowledge rotation training system for medical professionals; and regularly evaluate public health knowledge and practices of healthcare professionals and managers.

\# Corresponding author: Xifeng Wu, xifengw@zju.edu.cn.

\footnotetext{
School of Public Health, Zhejiang University, Hangzhou, China; 2 The Second Affiliated Hospital, Zhejiang University School of Medicine, Hangzhou, China; ${ }^{3}$ National Institute for Data Science in Health and Medicine, Zhejiang University, Hangzhou, China.
}

Submitted: March 16, 2020; Accepted: March 18, 2020

\section{REFERENCES}

1. World Health Organization. Coronavirus disease 2019(COVID-19) Situation Report - 52. Geneva, Switzerland: World Health Organization; 2020. https://www.who.int/docs/default-source/coronavi ruse/20200312-sitrep-52-covid-19.pdf?sfvrsn=e2bfc9c0_2. [2020-3-12].

2. Report of the WHO-China Joint Mission on Coronavirus Disease 2019(COVID-19), February 16-24, 2020. https:/www.who.int/ 
docs/default-source/coronaviruse/who-china-joint-mission-on-covid-19final-report.pdf. [2020-02-28].

3. Wang C, Horby PW, Hayden FG, Gao GF. A novel coronavirus outbreak of global health concern. Lancet 2020;395:470-3. http://dx.doi.org/10.1016/S0140-6736(20)30185-9.

4. Wu XF, Xu XL, Wang XC. 6 lessons from China's Zhejiang Province and Hangzhou on how countries can prevent and rebound from an epidemic like COVID-19. https://www.weforum.org/agenda/2020/03/ coronavirus-covid-19-hangzhou-zhejiang-government-response/? from=singlemessage. [2020-3-13].

5. China TCPsGotPsRo. The Regulation on Public Health Emergency 2003. http://www.gov.cn/zwgk/2005-05/20/content_145.htm. [20203-13]. (In Chinese).

6. China TCPsGotPsRo. Measures for the administration of information reporting on the monitoring of public health emergencies and epidemic situation of infectious diseases. 2003. http://www.satcm.gov.cn/ bangongshi/zhengcewenjian/2018-03-24/1143.html. [2020-3-13]. (In Chinese).

7. NHFPC. Guide on prevention of human infection of swine flu 2009.http://www.gov.cn/zwgk/2009-04/30/content_1300891.htm. [2020-3-13].

8. NHFPC. Guide on Preventing Nosocomial H7N9-Infection. 2013. http://www.gov.cn/gzdt/2013-04/08/content_2372212.htm. [2020-313]. (In Chinese).

9. NHC. China health statistics yearbook 2019. Beijing: Peking Union Medical College Press. 2019; p. 102-103. (In Chinese)

10. NHC. China health statistics yearbook 2019. Beijing: Peking Union Medical College Press. 2019; p. 28. (In Chinese)

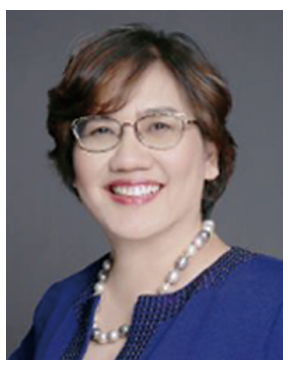

Xifeng Wu, MD, PhD

Dean, School of Public Health, Zhejiang University

Vice President, The Second Affiliated Hospital, Zhejiang University School of Medicine

Director, National Institute for Data Science in Health and Medicine, Zhejiang University 